\title{
Des microbes à vocation gandhienne dans un digesteur à biogaz
}

Digestion anaérobie et évolution de la recherche sur la technologie du biogaz

Dhruv Raina et Hoysala N. Chanakya

Traducteur : Olivier Morice et Valentine Leÿs

\section{OpenEdition}

\section{Journals}

Édition électronique

URL : https://journals.openedition.org/tc/8481

DOI : $10.4000 /$ tc. 8481

ISSN : $1952-420 \mathrm{X}$

\section{Éditeur}

Éditions de l'EHESS

\section{Édition imprimée}

Date de publication : 6 juin 2017

Pagination : 154-175

ISBN : 978-2-7132-2707-3

ISSN : 0248-6016

Référence électronique

Dhruv Raina et Hoysala N. Chanakya, "Des microbes à vocation gandhienne dans un digesteur à biogaz », Techniques \& Culture [En ligne], 67 | 2017, mis en ligne le 06 juin 2019, consulté le 29 septembre 2022. URL : http://journals.openedition.org/tc/8481 ; DOI : https://doi.org/10.4000/tc.8481 


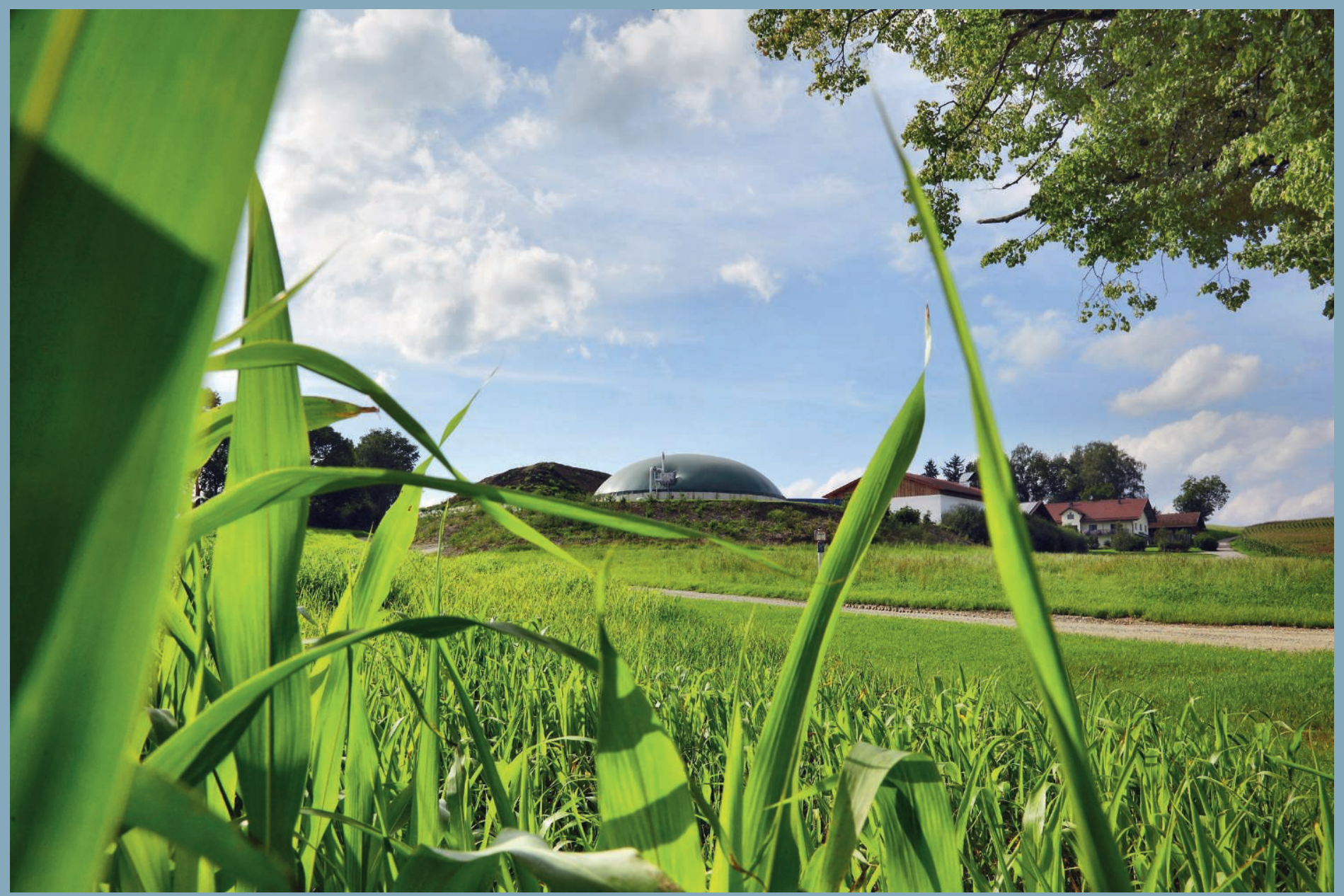




\title{
Une histoire des contingences technologiques
}

\author{
Digestion anaérobie et évolution de la recherche \\ sur la technologie du biogaz
}

Depuis quelque temps déjà, les historiens évoquent la possibilité d'intégrer l'histoire des techniques dans une théorie plus vaste de l'évolution biologique (McClellan \& Dorn 1999). Parallèlement, ces vingt dernières années, sont apparues diverses questions interdépendantes, qui mêlent conceptualisation théorique sociale (celle des chercheurs qui travaillent dans les cadres critiques des études du développement) et études critiques des sciences et technologies. Cette question sera traitée plus en détail dans ce qui suit. On notera néanmoins d'emblée que l'un des défis essentiels était de conceptualiser, aisément, la relation entre innovation technologique et développement social. D'un autre côté, un deuxième fondement théorique, plus ancien peut-être, qui a été plus ou moins remanié avec l'invention du concept de «technoscience » - concept aujourd'hui assez répandu - concerne la façon d'envisager la relation entre la science et la technologie comme étant mutuellement constitutive (Forman 2007, Narashima 2003). Le succès mitigé de certains systèmes technologiques d'une part et l'accumulation d'éléments attribuant la survenue de diverses catastrophes à la technologie d'autre part ne sont que les signes de la relation très complexe qu'entretiennent l'évolution technologique et le changement social. Dans l'un des premiers travaux de doctorat sur l'étude d'un laboratoire, Shiv Visvanathan souligne la nécessité d'intégrer la sociologie du développement dans la sociologie des sciences et de la technologie (Visvanathan 1985). Beaucoup a été fait depuis pour préciser la relation qu'entretiennent les Science and Technology Studies (STS) avec les perspectives critiques du développement et des études sur le développement (Harding 2011).

Par conséquent, en dépit de la relation triadique entre science, technologie et développement social, chacun de ces domaines se présente comme une forme exemplaire de savoirs, d'orientations et de pratiques possédant une autorité morale et une légitimité politique. Les 
deux perspectives critiques des STS et des études du développement sont au fait de la vie sociale des systèmes technologiques et inversement, ce qui déstabilise les représentations linéaires de l'évolution technologique et du développement programmé qui suit des « chemins de dépendances » (Smith 2009 : 9). La déstabilisation des représentations et des cadres conceptuels traditionnels est rendue possible par l'historicisation de la relation entre la science, la technologie et les études et cadres du développement (ibid.).

Dans le présent article, nous examinons le modelage social du programme d'un groupe de recherche de l'IISc (Institut indien des sciences, Bangalore). Ce groupe, qui se consacre à l'étude des frontières entre science et technologie, a dû, au cours d'une période de crise de légitimité du système de recherche scientifique, naviguer entre des régimes high tech et low tech divers et multiples, qui, en définitive, se sont révélés être les éléments d'un même continuum. Dans un autre article, l'un de nous s'était intéressé à la genèse du programme de recherche de la Cellule de l'IISc pour l'application de la science et de la technologie aux zones rurales (ci-après «ASTRA ») ${ }^{1}$ (Raina 1993). Le présent article ausculte les généalogies qui ont conduit au programme de développement du biogaz entrepris à l'IISc ainsi que l'évolution ultérieure de ce programme. Plus précisément, il ne s'agit pas de retracer l'histoire de la recherche sur le biogaz dans son ensemble, mais plutôt de décrire les chemins qui ont mené à la mise en place d'un programme de recherche unique. Si les travaux, pour une très large part, ont été menés dans les laboratoires d'ASTRA, ils ont aussi été marqués par des échanges réciproques sur les savoirs et les pratiques d'artisans et d'entreprises installés en milieu rural ainsi que par des mouvements sociaux, qui tous, à un moment ou à un autre, ont tiré profit de l'expertise high tech de l'IISc, expertise qu'ils ont, en retour, enrichie de leurs propres connaissances.

Ce qu'il y a de vraiment remarquable dans le fonctionnement de ces groupes, c'est l'interfoliation facile que l'on observe dans leurs façons de pratiquer des ensembles technologiques appartenant à des constellations et à des contextes temporels, culturels et institutionnels distincts. Comme le soulignent Latour et Serres, dans ce domaine, on ne saurait parler de démarcation nette entre le traditionnel et le moderne. Peut-être observe-t-on plutôt un continuum, qui serait en permanence en mouvement (Serres \& Latour 1990). L'histoire du concept de savoir indigène ou de ses formes externalisées que sont notamment la science et la technologie indigènes est un exemple typique de ce phénomène. Dans le prolongement de l'adoption du communiqué de Bangalore, comme on l'a avancé ailleurs, nous assistons, dans le cadre des travaux de l'UNESCO visant à élaborer un nouveau contrat social pour la science, à une normalisation de la science et de la technologie dites indigènes. Ce processus est à son apogée dans l'intégration des savoirs indigènes et locaux qui préside au document de vision de l'UNESCO pour 2030 (Raina 2016) ${ }^{2}$. Cela étant, nous pouvons revoir notre conception des mouvements technologiques intermédiaires, alternatifs et appropriés de la fin des années 1960 et des années 1970 et considérer qu'ils constituent la première étape d'une rencontre redéfinie entre la science ou le « savoir indigène ». La différence par rapport à la rencontre avec la technologie indigène des vingt dernières années est que le moment présent problématise très consciemment le concept d'évolution séquentielle, qui est le signe d'une progression constante de la technologie. 
Le présent article examine la généalogie d'un programme de recherche dans le domaine de la production de biogaz à l'IISc et identifie plusieurs généalogies scientifiques et technologiques connexes, leurs vies sociales et la circulation des idées technologiques afférentes dans un pays, une culture et un environnement. Ce faisant, nous nous attachons à montrer que la technologie de production de gaz de gazogène et de gaz de houille pour l'éclairage de rue apparue en Angleterre s'est propagée en Inde entre les années 1930 et 1950, a été fondamentalement remaniée dans les années 1980 et 1990, puis a migré vers la France et la Suisse dans la première décennie du xxi siècle. Autrement dit, si la circulation des idées scientifiques a fait l'objet de maints débats dans l'histoire des sciences, la mutation constante de l'évolution technologique vient peut-être limiter la façon dont les universitaires dégagent les contours du mouvement des idées et des pratiques technologiques. Le processus de circulation, la capacité constitutive de la technique et des systèmes technologiques à travestir les catégories philosophiques et sociothéorétiques des métathéoriciens est, de fait, une difficulté conceptuelle extrêmement préoccupante.

On sait que les inventeurs, les innovateurs et les personnes impliquées dans la promotion des nouvelles technologies influent sur la définition des priorités passées, présentes et futures qui déterminent les efforts d'investissement technologique. Mais, dans une perspective métahistorique, cela signifie qu'ils jouent en définitive un rôle direct ou indirect dans le réalignement des généalogies du passé en conférant un schème téléologique qui conduit tant au contemporain qu'au futur. Pour contourner la conception triomphaliste de la technologie, avec ses affirmations ancrées dans quelque variante de la théorie de la modernisation, Edgerton propose de substituer les histoires axées sur l'innovation à une histoire de la technologie centrée sur l'usage. Prenant appui sur l'argument de Latour et Serres mentionné plus haut, nous nous intéressons ici à une historiographie qui met en relation une multitude de lieux et de technologies anciennes et nouvelles, en nous affranchissant des présupposés des théories du déficit et en rejetant les affirmations des tenants de l'innovation (Edgerton 2007 : xI). Les modèles du déficit sont ancrés dans des conceptions modernistes de la technologie et s'appuient sur la distinction entre le traditionnel et le moderne, distinction que nous cherchons à dépasser. L'une des raisons d'être de notre entreprise est que la technologie, comme le temps, a été brassée, ce qui donne à penser que l'ancien, le médiéval et le nouveau coexistent dans le moderne. En conséquence de quoi, les anciennes technologies de la représentation moderniste disparaîtraient ou deviendraient obsolètes et seraient donc condamnées. Mais en réalité, « les technologies ne se contentent pas d'apparaître, elles disparaissent et réapparaissent et se panachent au cours des siècles » [Edgerton op. cit. : xiI]. Autrement dit, les technologies et les programmes de recherche entrent souvent en hibernation, puis sont influencés, dans leur longue évolution, par des facteurs sociaux divers et multiples.

Dans de nombreuses régions d'Asie du Sud, les deux décennies qui s'étendent des années 1970 aux années 1990 ont été le théâtre d'un nouvel investissement néogandhien dans la science et la technologie, à mesure que de nouveaux mouvements sociaux prenaient de l'essor partout en Inde et que l'optimisme « nehruvien » déclinait (Raina 1997 ; Visvanathan 
1997 ; Raina, Chowdhury \& Chowdhury 1997 ; Seshadri 1999). Pendant cet interlude, les mouvements et programmes de technologies alternatives ont assurément tiré leur miel et leur inspiration d'une lecture de Gandhi, que lui-même aurait ou non reconnue. Amulya Kumar Narayana Reddy, éminent électrochimiste devenu analyste des politiques énergétiques, a défini des critères pour aider au choix des technologies; ces critères ont orienté les priorités et les programmes de recherche de la cellule ASTRA (Reddy et al. 1988) ${ }^{3}$. Et tout en soulignant l'importance de cet outil stratégique, il faut être conscient que la dichotomie Ganghi-Nehru, perçue comme une dichotomie entre le luddisme et le techno-évangélisme ou entre le traditionnel et le moderne, est tout aussi réductrice et indéfendable (Guha 2007). Dans un cas comme dans l'autre, il serait intéressant d'examiner comment ces assemblages technologiques alternatifs (Müller \& Schurr 2016) ${ }^{4}$ - si l'on renonce au terme «système » pour les désigner - ont été façonnés dans le cadre nehruvien-gandhien. Plus tard, avec l'achèvement d'une phase et le commencement d'une autre, on peut s'interroger sur les connotations qu'acquiert l'expression « technologie durable» dans le cadre néolibéral et dans le cadre néogandhien.

Une dernière question retiendra notre attention. Au cours de divers entretiens avec des scientifiques associés à ces programmes, il est apparu important de comprendre comment, à l'instar des idées scientifiques, les technologies hibernent ou sont mises de côté. Comprendre pourquoi elles sont remises à l'honneur, pourquoi une idée traditionnelle et datée amorce un nouveau cycle de développement et prend une trajectoire qui était parfois insoupçonnée au moment de sa mise en sommeil. En fait, on pourrait voir dans le mouvement des technologies alternatives une tentative d'incorporer des technologies indigènes dans des systèmes technologiques contemporains, une manière de redynamiser ces technologies, qui, fussentelles mises en réserve, ne sont pas pour autant obsolètes. L'approche historique centrée sur l'usage contourne les limitations de l'approche tournée vers l'innovation, et offre un équivalent conceptuel à l'idée lakatosienne de la mise en sommeil des théories scientifiques (Edgerton op. cit.). Ce pas de côté dans l'historiographie du présent nous oblige à revoir notre compréhension du déchet, de l'obsolescence et de l'oubli. Dans A History of the World in 100 Objects, MacGregor rappelle ce qui suit :

« Les objets qui sont jetés ou perdus nous en disent autant sur le passé que bon nombre d'autres minutieusement conservés pour la postérité. Les objets ordinaires du quotidien, mis au rebut il y a longtemps, ont des récits à raconter parmi les plus importants de l'histoire de l'humanité » (MacGregor 2012 : 22).

L'objet du présent article concerne l'utilisation du biogaz en tant que déchet exploité à des « fins utiles » et, parallèlement, le caractère contingent de l'évolution technologique lorsque des technologies obsolescentes sont ressuscitées pour suivre des trajectoires qui n’avaient pas été anticipées.

Le caractère contingent du développement technologique se manifeste dans les applications non prévues qui se présentent dès lors que l'on étudie la technologie dans sa dynamique. Les technologies ont in fine de multiples usages, qui dépendent des ressources locales et de divers 


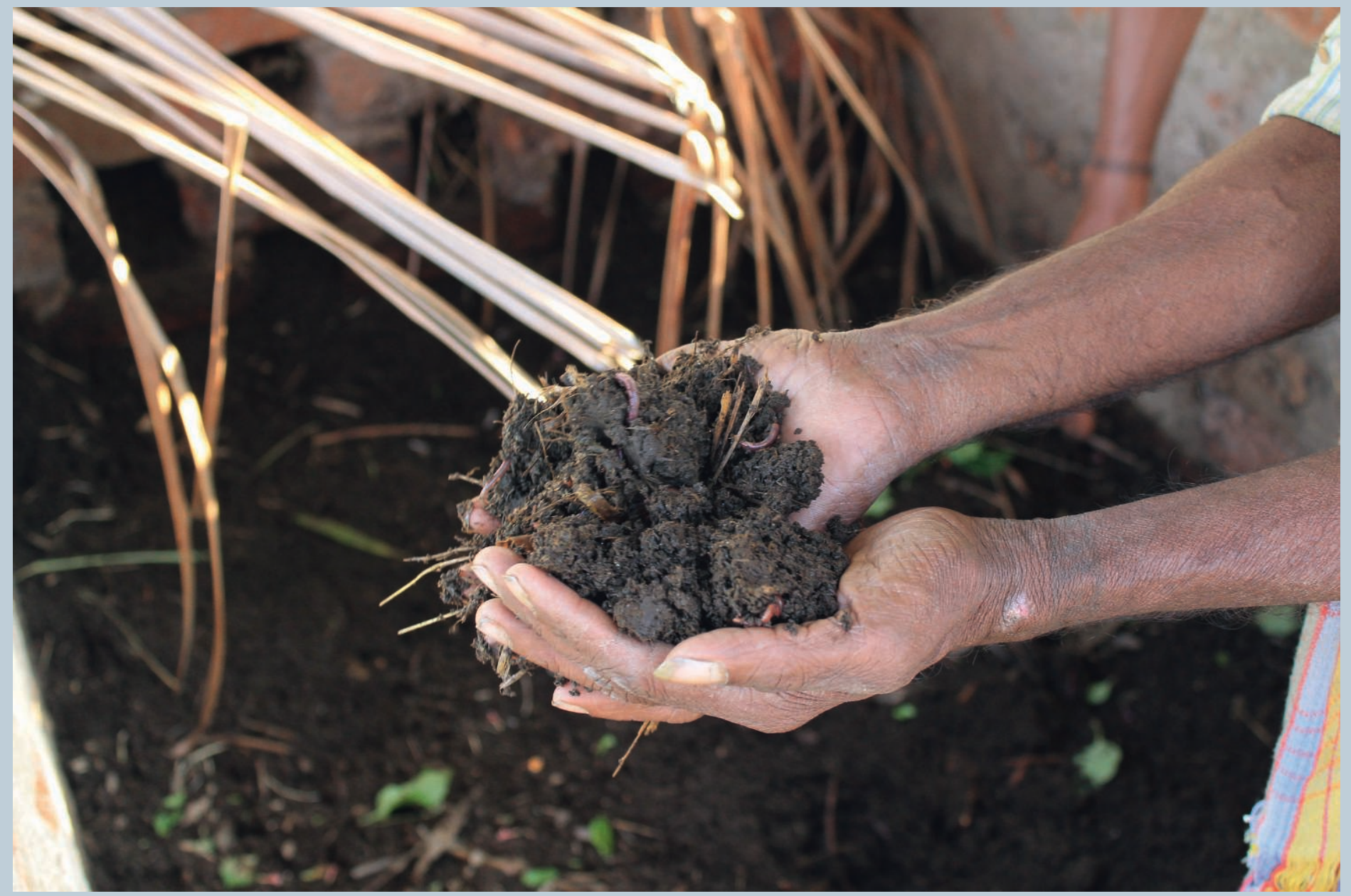

7. Le fumier des bovins est mixé avec de l'eau et inséré dans le biodigesteur. 
facteurs notamment culturels. De ce fait, le récit de l'invention, ou l'histoire de la technologie centrée sur l'innovation, est remis en question par la métaphore d'une image rhizomique complexe, dans laquelle un processus de dendrification est à l'œuvre autour d'une multitude de nœuds. L'histoire de la technologie du biogaz que nous nous proposons de retracer est de cet ordre, si ce n'est qu'elle se déroule sur une seule branche de ce réseau.

Nous pouvons aussi expliquer ces phénomènes en invoquant l'approche par cycle technologique. Prenons l'exemple d'une nouvelle technologie qui recueillerait une large adhésion grâce à l'une de ses nombreuses applications. Lorsque la première application ou utilisation pour laquelle cette technologie a été diffusée à l'origine parvient à son terme, cette même technologie peut être adaptée très rapidement, servir de base à une deuxième application et être remise sur le « marché ». À cette étape, la période de gestation est très courte et les technologies n'ont pas besoin de suivre les processus complexes de validation, d'acceptation par les consommateurs et d'internalisation. Seule la partie nouvelle ou ajoutée de la technologie subit le retard d'internalisation.

Par ailleurs, une technologie, un dispositif technologique ou une application, de manière générale un assemblage technologique, aura été mis au point pour une utilisation finale bien précise, alors qu'il renferme potentiellement d'innombrables autres applications connexes et non prévues qui n’ont jamais été explorées ou encouragées avec suffisamment de vigueur parce que non envisagées sous l'angle de l'invention. Du reste, s'agissant de la question des assemblages, les obstacles, perçus comme tels, à la diffusion ou à la mise au point d'une technologie peuvent souvent être évités en empruntant des trajectoires de développement parallèles. Par exemple, les réticences liées à la production de biogaz en bouteille à partir de déchets urbains solides et à l'utilisation de ce carburant dans les transports en commun et les véhicules de voirie ont été surmontées par voie de justice en insistant sur la nécessité de mettre en place des transports moins polluants. Plus récemment, l'utilisation du GNC (gaz naturel comprimé) a même été autorisée. Par sa décision, le tribunal a fait pression sur les chercheurs pour qu'ils conçoivent un biogaz purifié (sans CO2) utilisable dans les transports urbains. Bien que le procédé ait été connu depuis plus d'un demi-siècle, le biogaz n'avait jamais été envisagé comme un carburant (Chawla 1986 : 96). Sans doute ne pouvons-nous pas écarter la dimension économique du problème (tarifs compétitifs, intérêts géopolitiques et stratégiques, etc.), qui est contrebalancée par une cosmopolitique de la survie fondée sur la nécessité absolue d'abandonner les carburants fossiles. En d'autres termes, après une période de résistance à toute activité méthanogène mettant en œuvre des biogaz, l'utilisation du biométhane trouve aujourd'hui sa justification dans le changement climatique et les avantages compétitifs (Johansson, Kelly, Reddy \& Williams 1993 ; Chanakya \& Balachandra 2012 : chap. 10 ; Chanakya \& Malayil 2012). Ces avancées latérales ou incrémentales se produisent aujourd'hui sans trop de retard. 


\section{Le contexte de développement des années 1960 et 1970}

Ce qui est frappant chez Reddy et ses associés, c'est la manière dont certains concepts gandhiens essentiels et certaines façons de voir le monde « depuis l'autre côté » sont imbriqués dans les programmes de développement technologique. Quand les valeurs sont-elles incorporées dans ces programmes? Est-ce avant le début du programme, ou la restructuration commencet-elle une fois le programme achevé ? Ces questions, tout en étant au cœur de l'étude des techniques et de la culture, étaient importantes dans le contexte des préoccupations plus vastes de Gandhi sur la place de la technologie moderne dans la vie contemporaine.

Si les années 1970 ont été une période de crise pour le développement et la modernisation (Elzinga \& Jamison 1981), en Inde aussi, ces années ont été marquées par une crise, celle du mandat nehruvien, qui a donné lieu à diverses interventions au niveau des institutions et de l'économie politique. La crise énergétique, de portée mondiale, a été l'événement le plus frappant de cette période, et les crises environnementales et écologiques, dans leur relation avec la crise énergétique, ont amené à repenser le développement, la modernité et l'industrialisme. Dans ce contexte, en Inde, le milieu des années 1970 a été marqué par un sentiment de déconnexion radicale entre la science, la technologie et l'industrie. Aussi les décideurs ont-ils remis en perspective le mandat nehruvien ainsi que la centralité de la science et de la technologie dans ce contexte (Raina 2003). Dans le nouveau paysage qui se dessinait, les mots en vogue étaient « développement » et " politique de développement », « relations sociales de la science » et "politique scientifique », et, dans les interstices entre "science et développement », les politiques « scientifiques et de développement » constituaient le terrain du nouveau discours.

En Inde, la pauvreté était généralisée : la théorie de la relance par le haut n’avait manifestement pas fonctionné et les stratégies de développement et les transferts technologiques n'avaient pas permis d'éradiquer ce fléau. Dans le monde de la recherche scientifique et universitaire, cette crise structurelle a pris la forme d'une crise de légitimité organisationnelle et institutionnelle (ibid.). De nouveaux mouvements sociaux sont apparus et quelques étudiants d'instituts scientifiques prestigieux ont migré vers ces nouveaux repères du paysage scientifique indien : mouvements de technologie alternative, mouvements de science du peuple, etc. Plusieurs ingénieurs scientifiques, chimistes et biologistes de l'IISc qui, dans la communauté scientifique, affichaient un engagement intellectuel « conventionnel » et jouissaient d'une réputation à la hauteur de cet engagement se sont regroupés et ont créé la cellule ASTRA, dont l'objet était d'étudier les problèmes de développement de l'État et plus particulièrement ceux des régions rurales du Karnataka.

Il va de soi qu'une telle cellule aurait été difficilement justifiable au sein d'un institut comme l'IISc, dont le mandat était de mener des travaux de recherche aux limites de la science. Les scientifiques de la cellule ASTRA, qui étaient hébergés dans l'IISc même, devaient absolument jouir d'une réputation sans faille dans leurs disciplines respectives pour pouvoir survivre aux éventuelles moqueries de collègues leur reprochant de choisir la facilité. Les circonstances ont 
joué en leur faveur : 1) la plupart d'entre eux étaient reconnus dans leur domaine ; certains étaient des chefs de file, et les autres se forgeaient une réputation; et 2) le directeur, Satish Dhawan, spatiologue de renom, avait imprimé sa marque sur le programme. Pourtant, le statut inférieur du « développement rural » dans la hiérarchie des sciences continuera de hanter les chercheurs associés à la cellule ASTRA. Ce qui n'a pas empêché l'unité d'exister au sein de l'institut. Elle était, comme je l'ai dit ailleurs, une île de doutes au milieu d'un océan de certitudes (Raina 1993 op. cit.).

La métaphore de la lune et du ghetto - une analogie post-Apollo - a souvent été utilisée pour faire taire ce genre de critiques. Mais à moins d'être en prise directe avec les technologies rurales, un praticien de la science aurait du mal à se laisser convaincre que les problèmes posés par ces technologies peuvent être aussi ardus que ceux qu'il a l'habitude de rencontrer. En l'occurrence, comme le dit l'adage, «moins on en savait, mieux on se portait ». Au sein de l'institut, la cellule ASTRA et Amulya Reddy demeuraient les voix de la science dissidente, la première avec discrétion, le second plus ouvertement (Reddy 1978b). Les membres d'ASTRA comprenaient la technologie comme un déterminisme technologique. Toutefois, leur entreprise s'appuyait sur certaines valeurs et idéologies, qui, bien que parfois expressément formulées, affleuraient souvent à peine à la surface de l'expression consciente. L'incapacité à formuler ces valeurs s'expliquait peut-être par l'influence de la culture technocratique, qui aurait jugé avec dédain des propos contraires à l'idée de neutralité de la technologie.

La critique du développement industriel qui a refait surface dans les années 1970 doit beaucoup aux écrits de Gandhi. L'ouvrage Small is Beautiful d'E. F. Schumacher (1975) en particulier reprenait l'idée selon laquelle la technologie industrielle à grande échelle ne s'accompagne pas nécessairement d'économies d'échelle ou d'une réduction des coûts unitaires. Le centre d'écriture de Gandhi met en scène les problèmes de l'Inde rurale ; l'économie gandhienne et le tabou de l'inceste ont défini les priorités et délimité le champ des études du groupe. Tels sont les principes qui ont été exposés avec force dans un article de Reddy devenu classique sur le choix des technologies. Ces critères ont servi d'indicateurs pour les programmes de recherche des membres de son équipe lorsqu'ils ont abandonné l'idée des « rectificatifs technologiques » et qu'ils sont sortis des laboratoires de l'IISc pour se rendre dans les villages du Karnataka (Reddy op. cit.). D'une certaine façon, ce que cette vision rejetait, c'était le postulat industriel selon lequel « les économies d'échelle justifient la production en série ». La prudence gandhienne à l'égard du consumérisme était désormais reformulée dans les travaux du groupe comme la possibilité d'envisager et de créer un futur viable (Gandhi 1922) 5 .

Il n'est pas très difficile de trouver les racines de la pensée gandhienne dans les critères applicables au choix de la technologie proposés par Reddy. Ces critères étaient les suivants : 1) La technologie répond-elle aux besoins des plus démunis ?2) La technologie mobilise-t-elle des ressources humaines locales? 3) La technologie est-elle respectueuse de l'environnement (Reddy 1978a)? Un quatrième critère concernant l'autonomisation des femmes au niveau local a été introduit à la fin des années 1980. On espérait que ces critères guideraient le développement de technologies propres à redynamiser l'économie villageoise. Quoi qu'il en soit, 
ces idées ont inspiré plusieurs mouvements et ONG présents dans l'État du Karnataka et dans les États voisins. La priorité mise sur la vie quotidienne en milieu rural a donné lieu à plusieurs programmes sur l'énergie et l'habitat particulièrement soucieux de facteurs environnementaux, cette préoccupation étant, à l'époque, un élément constitutif des travaux du groupe (Reddy op. cit. 1978b).

Il y avait dans ce contexte deux présupposés technologiques majeurs. Le premier était que les technologies dites traditionnelles avaient évolué au fil des siècles et été maintes fois adaptées dans des contextes et des environnements locaux; ces connaissances technologiques et les savoir-faire associés étaient transmis, tel du matériel génétique, de génération en génération (Reddy 1978a). L'idée connexe était d'utiliser, autant que possible, ces connaissances et ces savoir-faire comme un moyen de produire de nouvelles technologies et de nouveaux systèmes, et peut-être ainsi d'atténuer les difficultés d'adaptation et d'assimilation. Cette vision des choses a incité les scientifiques à ne plus régler les problèmes de technologie en recourant à des solutions technologiques généralisables, ce qu'ils avaient tendance à faire parce que cela faisait partie de leur socialisation cognitive (Reddy op. cit. 1978b). L'autre facteur qui a peut-être contribué à cette vision des choses a été la prise de conscience que les problèmes des régions rurales venaient des rectificatifs technologiques qui étaient imposés sans distance critique à des contextes auxquels ils ne s'appliquaient pas.

\section{Ethnographie du programme biogaz de l'IISc}

Nous cherchons maintenant à mettre en évidence les généalogies de la recherche sur la biogazification à l'IISc, et ce en nous appuyant sur une analyse ethnographique, plusieurs entretiens et une étude bibliographique.

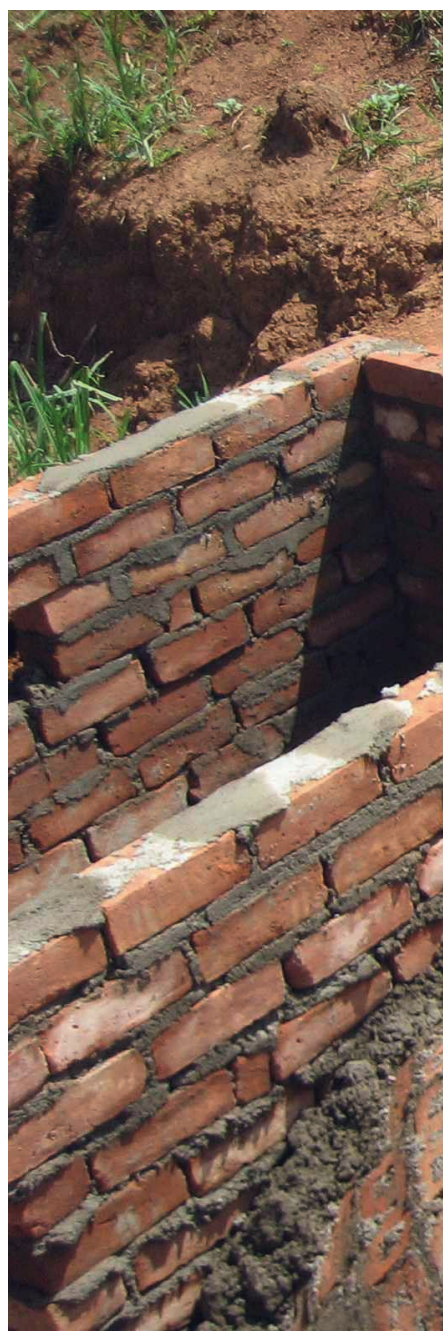
Notre objectif est aussi d'attirer l'attention sur la richesse des matériels analysés. Premier institut de recherche scientifique indien, l'IISc est créé en 1909 par un accord triangulaire original entre le groupe industriel de Jamsetji Tata, le gouvernement impérial et l'ancien État du Mysore (Subbarayappa 1992). Les fondateurs prennent pour modèle l'Université Johns Hopkins de Baltimore, à l'intersection entre le monde de la connaissance et celui de l'industrie (Subbarayappa 1992). Au début des années 1920, l'institut est doté d'un estimable programme de recherche en microbiologie et biochimie, comme en atteste un historique interne détaillé du département de biochimie. Pendant cette décennie, deux scientifiques de l'institut, Fowler et Joshi, publient un article important (Fowler $\&$ Joshi 1921) sur la digestion anaérobique des déchets agricoles (Subbarayappa op. cit. : 


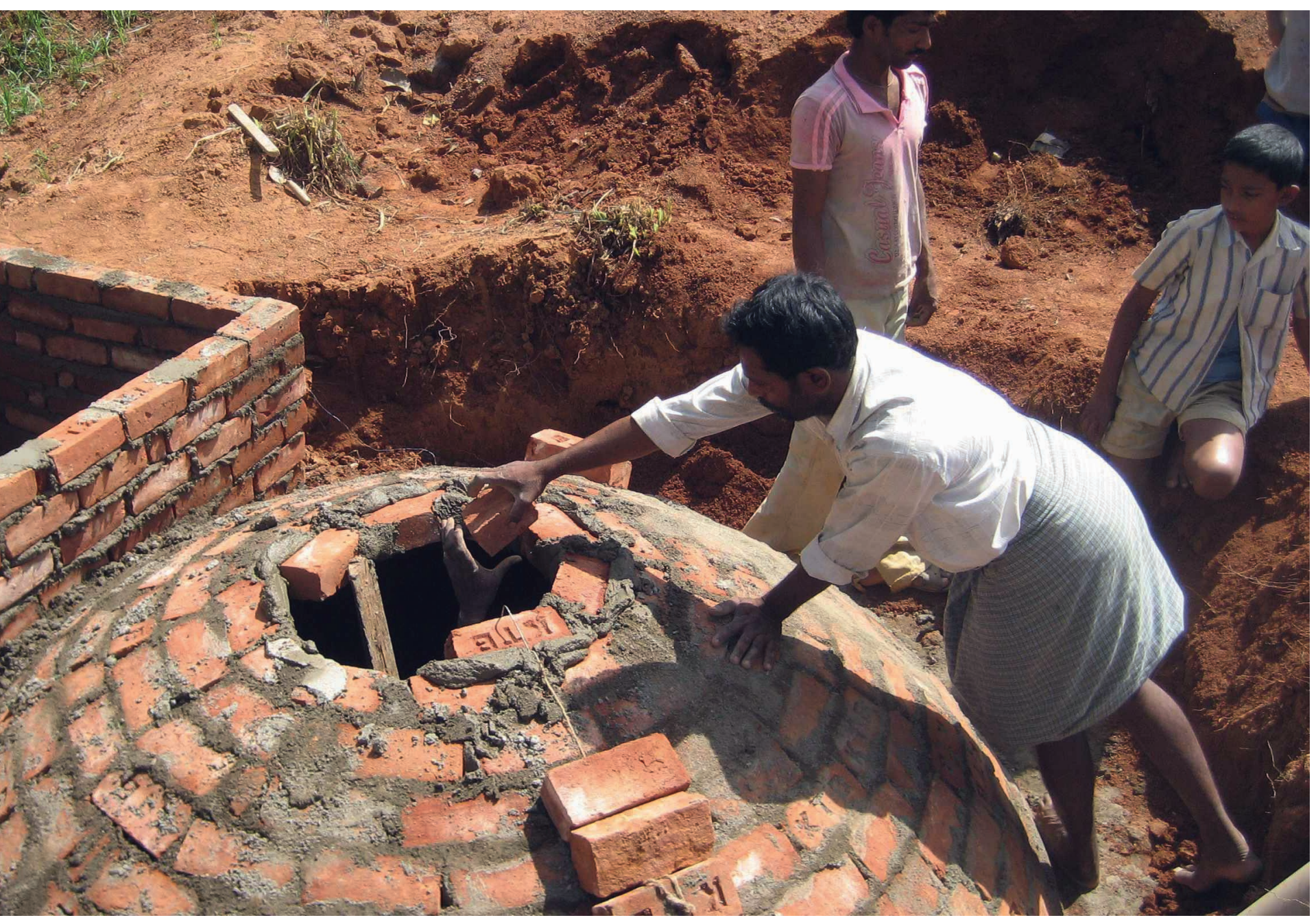

89-90) ${ }^{6}$. L'objectif est manifestement de parvenir à fixer l'azote dans le sol pour augmenter le rendement des terres, la production de biogaz venant en prime (Chawla op. cit. : 8).

C. N. Acharya, chercheur à l'IISc, commence également à travailler sur la dégradation de la cellulose végétale et met sur pied l'un des premiers programmes de digestion anaérobique des déchets agricoles. Ce programme consiste, entre autres, à évaluer la quantité d'azote fixée ainsi que le volume de gaz produit (Acharya 1935). Il publie ses travaux alors qu'il est en poste à Rothamstead, mais ne mentionne pas, dans ses articles, la quantité de gaz inflammable produite. Il retourne à l'IISc une première fois vers 1939-1940, puis se rend à l'Institut indien de recherche agricole, où l'utilisation des fertilisants synthétiques n'en

Réservoir à biogaz en construction

Recycler des déchets domestiques et le fumier animal, améliorer les conditions de vie des fermiers, de leurs familles... : I'utilisation du biogaz contribue également à protéger les forêts indiennes.

District de Hassan, État du Karnataka (Inde) 
est qu'à ses débuts, leur prix étant, du reste, encore exorbitant (Acharya 1940ab). Acharya poursuit ses travaux sur le compost et sur la fixation et la conservation de l'azote dans le sol. L'inconvénient du compostage traditionnel à l'air libre est qu'il conduit à l'évaporation, dans l'atmosphère, de l'azote contenu dans les déchets agricoles; le compostage anaérobique, en revanche, permet de fixer et de conserver l'azote dans le sol (ibid.). Les premiers brevets sur le compostage anaérobique de la bouse de vache sont déposés par N. V. Joshi en 1940, puis par Jasubhai Patel (1949-1952) et Acharya (1949) (Chawla op. cit.). La production de méthane inflammable est alors connue, mais aucune recherche n'est menée pour augmenter le rendement à ce stade.

À l'époque, plusieurs technologies de gazogène sont disponibles dans l'État du Mysore (aujourd'hui Karnataka) (Raghunandan \& Chanakya 2004). Les rues des villes européennes sont déjà éclairées au gaz et, à Londres, on utilise du gaz de gazogène, du gaz à l'eau ou du gaz de houille. En 1903, l'éclairage des rues à base de gaz de gazogène ou de gaz de houille arrive dans les villes du Mysore. Dès 1897, à Exeter (Angleterre) et à Bombay, on envisageait déjà de produire du gaz d'éclairage à partir d'excréments humains ou de déchets. De fait, cette année-là, une usine avait été construite dans la léproserie de Matunga, à Bombay. Mais dans les années 1930, au Royaume-Uni, reprenant l'idée de la production de gaz d'éclairage dans les usines de gaz de houille, les chercheurs conçoivent un modèle stable de chambre de réaction, qui est de plus en plus souvent évoqué comme substitut au charbon pendant la guerre. Dans l'État du Mysore, des bus sont alimentés par ce type de gazogène.

Cela étant, dans l'Inde post-indépendance, une coopérative publique dotée du statut de commission, reprend les idées de Gandhi sur la redynamisation du secteur artisanal traditionnel, et s'intéresse aux usines de production de biogaz à partir de bouses. Les digesteurs anaérobiques sont désormais utilisés pour produire du gaz à des fins multiples et variées (Abbasi, Tauseef \& Abbasi, 2012 : chap. 2). Avec l'arrivée des fertilisants, la fixation de l'azote dans le sol n'est plus aussi essentielle. Trois nouveaux prototypes d'usines de biogaz sont bâtis et brevetés sous l'appellation Gramalaxmi (I et peut-être II et III). Ce nom, qui signifie déesse de la richesse du village, a son importance. Les premiers modèles des chambres de réaction de ces usines ressemblent à ceux des usines de gaz de houille et de gaz de gazogène; ils intègrent des contrepoids, des poulies et pléthores d'autres mécanismes. Le dernier modèle est plus simple (Desai \& Biswas 1945, Chawla op. cit. : 40).

Pourquoi la commission KVIC (Khadi and Village Industries Commision) décide-t-elle d'investir dans le biogaz et pourquoi l'excrément bovin est-il toujours valorisé ? Depuis très longtemps, les galettes de bouse séchées sont utilisées sur tout le territoire et dans de nombreuses autres régions du monde comme combustible pour la cuisine. La commission KVIC persuade Jasubhai Patel de breveter le prototype de l'usine de biogaz pour l'ensemble du pays. À l'instar de la vache sacrée, l'usine de biogaz est donc canonisée ; la promotion du biogaz commence dans les années 1960. Un agronome du nom de Desai et un fermier américain publient une série d'articles dans les revues Farmer's Digest et Indian Farming, combinaison efficace entre un scientifique et un praticien, qui diffusent tous deux une 
technologie parvenue à maturité (Chawla, Laura \& Idnani 1969 : 1040-1043 ; Idnani \& Varadarajan 1974). Mais avant d'utiliser le biogaz pour la cuisine et/ou l'éclairage sur l'ensemble du territoire, il faut d'abord et avant tout examiner la question des usines de production et de leur étendue. À cet égard, il importe de noter qu'au moment où ces questions se posent, l'économie mondiale n’a pas encore traversé la crise énergétique, qui n'éclatera que quelques années plus tard. Il semble ici que des contraintes très locales aient créé des opportunités qui pouvaient peut-être être concrétisées grâce au biogaz. À cette époque, l'utilisation des pompes à eau augmente de façon exponentielle, en particulier dans les terres arrosées par les pluies et non irriguées. Le diesel fait cruellement défaut même avant la crise énergétique, et l'on commence à envisager la possibilité de construire des moteurs à combustible mixte pour faire tourner les pompes. Pendant cette courte période sont publiés, dans diverses revues, quelque trente articles scientifiques rendant compte d'expériences sur des moteurs à combustible mixte (dont un gaz inflammable), suffisamment puissants pour entraîner des pompes à eau ${ }^{7}$.

Pendant cette phase, qui s'étend de 1965 à 1980, un certain nombre d'expériences sur des usines de biogaz sont menées. Plusieurs modèles voient le jour, dont certains sont le fruit de travaux visant à comprendre les mécanismes de la biogazification en vue de maximiser le rendement de biogaz obtenu pour un volume de fumier liquide donné (Chawla et al. op. cit., Idnani \& Varadarajan op. cit.). D’autres usines sont conçues par des ingénieurs et des bricoleurs qui, considérant que le mécanisme biochimique sous-jacent est déjà bien connu, ne se soucient pas autant du processus de digestion anaérobique de la biomasse (Sathianathan 1975, Reddy \& Subramanian 1979, Khandelwal \& Mahadi 1986).

En 1974, l'IISc lance un programme de recherche sur le biogaz, dont les différentes tâches sont réparties entre généticiens, ingénieurs et microbiologistes (Raina 1993 op. cit.). Les trois groupes ambitionnent d'augmenter l'efficacité et le rendement de conversion de la biomasse. Le premier travaille sur la conception du réacteur, le deuxième sur la cinétique chimique et la biochimie du processus, et le troisième sur l'efficacité et l'acceptabilité du mécanisme dans son ensemble. Les concepteurs du réacteur et les biochimistes et généticiens doivent travailler en collaboration (ibid. ; Rajabapaiah, Ramanayya, Mohan \& Reddy 1979 ; Rajabapaiah, Chanakya \& Reddy 1981).

Puis des agronomes rejoignent l'équipe et les efforts sont portés sur la diversification des matières biodégradables utilisées. Il s'agit de passer de la bouse de vache à d'autres matières végétales (Chanakya, H. N., Borgaonkar, S., Rajan, M. G. C. \& G. Meena 1992). Au début des années 1980, une équipe de scientifiques de l'IISc conçoit et construit des usines de biogaz dans des villages du Karnataka. Cette équipe est composée de chimistes, de microbiologistes, d'ingénieurs mécaniciens, d’ingénieurs du génie chimique, d'agronomes et d'agents de vulgarisation agricole. On prend alors brusquement conscience que la technologie aussi a une vie sociale, qui, peut-être, joue un rôle majeur. Les villageois pouvaient apporter des galettes de bouse à l'usine. Le gaz était distribué à tous les foyers du village à parts égales, mais la quantité de fumier liquide décomposé destinée à la 
fertilisation des terres ne pouvait être fournie qu'à proportion du volume de bouse apporté par chaque foyer (Rajabapaiah et al. 1981 op. cit.). Petit à petit, la vie du village s'organise autour de toutes sortes de registres dans lesquels sont consignées les heures de service des employés assurant le bon fonctionnement des usines, le chargement des bouses ou encore la redistribution du fumier liquide. Ces matières, qui ont toujours été considérées comme des déchets, ont désormais leur place dans l'économie monétaire. Cette mutation rapide fait naître de nouvelles préoccupations et des économistes s'invitent dans le débat sur l'économie du biogaz (Raina 1993 op. cit.). Cette contribution du groupe pourrait être anticipée par les économistes et par d'autres, estimant que le village devait recevoir une compensation pour les « déchets» dont ils faisaient don à l'usine.

Comme on envisage alors de construire de nouvelles usines de biogaz, les questions de volume, d'échelle et de disponibilité du fumier gagnent en importance. Le réacteur (ou l'usine) est prévu pour une production de gaz de 1,4 m3 par jour, ce qui requiert, au niveau du village, un volume minimum de fumier. Dans la zone semi-aride entourant la ville de Bangalore, la ressource n'est pas suffisante pour répondre à la demande des usines. Cet écart ressort des calculs effectués par la commission KVIC concernant la quantité de bouse produite par tête de bétail (soit $15 \mathrm{~kg} / \mathrm{jour}$ ). Ce chiffre n'a jamais été validé dans le sud du pays. Les valeurs et les estimations de la commission reposent sur des mesures réalisées sur des animaux en tabulation. Or les mesures réelles effectuées sur le terrain révèlent que les animaux paissant en plein air produisent $4 \pm 2,8 \mathrm{~kg} / \mathrm{jour}$, la marge d'erreur correspondant à la quantité de fourrage disponible (faible en été, et élevée pendant la mousson) (Chanakya $\&$ Balachandra op. cit.).

Dans le village de Pura, les besoins en matière de cuisson semblaient ne jamais être satisfaits, notamment en raison des pratiques culinaires. Ce problème se posait dans les régions où le nombre de bêtes fournissant la quantité de bouse requise était plus important. Lorsqu'il arrive à l'IISc, Howard Gellar, membre du groupe de recherche sur l'énergie de l'Université de Princeton, commence à travailler sur l'efficacité et le rendement des fourneaux de cuisine (Gellar 1982, Reddy \& Prasad 1997). Une fois les diverses contraintes prises en compte et la conception et l'exploitation des usines stabilisées, il apparaît que le gaz produit est tout juste suffisant pour répondre aux besoins de cuisson d'une famille pour un seul repas. Il ressort d'un rapport sur les travaux du groupe qu'il était, semble-t-il, plus réaliste d'utiliser le gaz pour éclairer les villages non encore électrifiés et pour alimenter les pompes d'adduction d'eau potable (Johansson et al. op. cit.).

Ces contraintes orientent le programme dans d'autres directions. Amulya Reddy, entre autres, demande à des ingénieurs généticiens de concevoir d'autres techniques de dégradation de la cellulose et d'augmenter le rendement du gaz d'un facteur dix. Les microbiologistes décident d'abandonner la bouse de vache au profit d'un large éventail de déchets végétaux et agricoles. Les ingénieurs chimistes sont invités à participer à la conception du réacteur, les problèmes d'efficacité de la production de gaz étant, estime-t-on, imputables aux différences de densité du fumier liquide dans ce dispositif (Chanakya \& Deshpande 


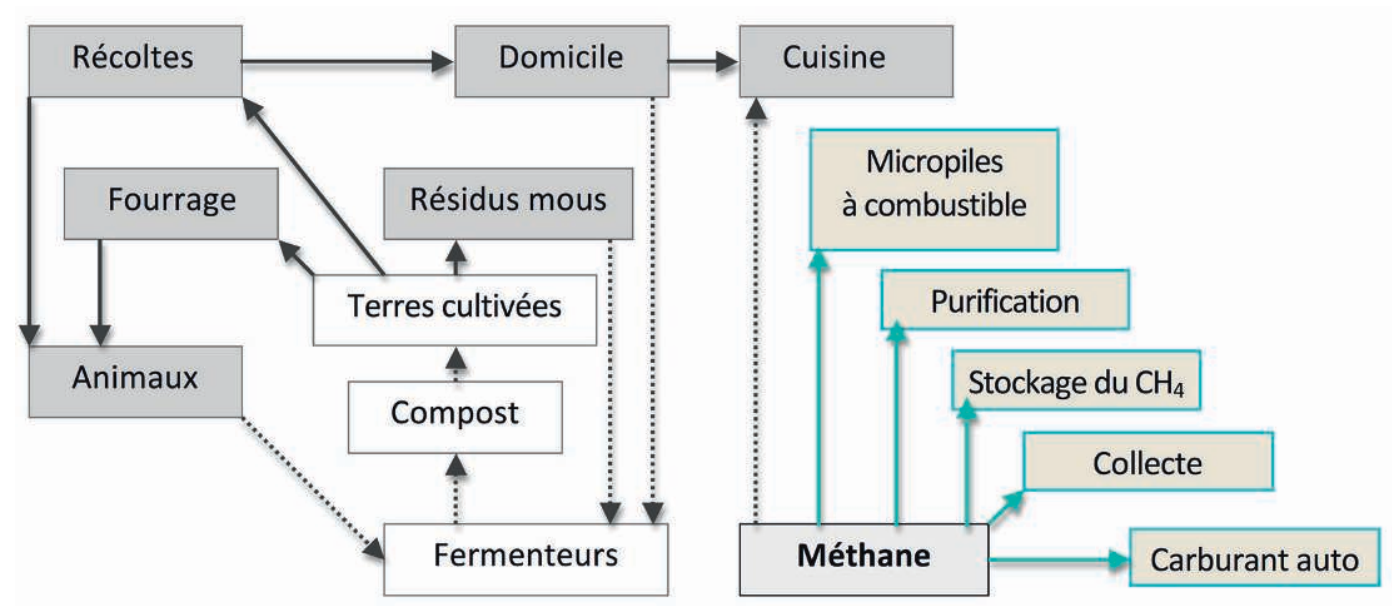

\begin{abstract}
4. Le diagramme fonctionnel ci-dessus illustre la nouvelle économie du méthane et l'intégration systémique du biogaz. Ce schéma est assez différent des applications limitées de la digestion anaérobique du biogaz dans un système d'approvisionnement en énergie renouvelable comme on l'envisageait dans les années 1970. Cela étant, cette évolution peut s'appréhender sous différents angles. On peut y voir, selon une conceptualisation téléologique proposée par l'un des participants au programme de recherche, une généalogie qui partirait de la recherche sur la digestion anaérobique pour aboutir à l'une des multiples branches de la recherche contemporaine sur le développement durable. Cette idée est illustrée dans le diagramme de la page suivante. (Source : Chanakya \& Balachandra, 2012 : 192)
\end{abstract}

1985). Disons pour faire simple que des techniques enzymatiques sont essayées pour décomposer la cellulose, mais que les bactéries produisant les enzymes disparaissent dans le réacteur. Autrement dit, les «super bactéries » pourraient efficacement décomposer la cellulose, mais elles ne sont pas suffisamment résistantes pour survivre dans l'environnement du réacteur. Le fumier liquide est soumis à deux étapes de digestion, mais cela ne règle pas le problème.

En 1984, Amulya Reddy et David Hall quittent le programme biogaz pour des réflexions plus générales sur l'énergie dans un monde durable. L'usine de biogaz de Pura est aujourd'hui à l'abandon et la cellule ASTRA a été transformée en un centre pour les technologies durables. Le programme sur les usines de biogaz à base d'excrément bovin s'était apparemment essoufflé et il fallait revenir aux sciences fondamentales et peut-être réinventer le concept de fermentation. Nous n'examinerons pas non plus ce processus, que nous réservons à un article ultérieur.

Vers la fin des années 1990, près de quinze ans après leur mise en sommeil, les usines de biogaz reprennent du service, moyennant certaines modifications. Cette partie de l'histoire doit être approfondie et nécessite de comprendre précisément comment et pourquoi, à un moment donné, des idées et des technologies remisées sont tirées de l'oubli. C'est à cette époque qu'une équipe d'ingénieurs de Narbonne découvre les usines de l'IISc de Bangalore. Une entreprise plus vaste a peut-être éveillé leur intérêt : des agences de développement sont 


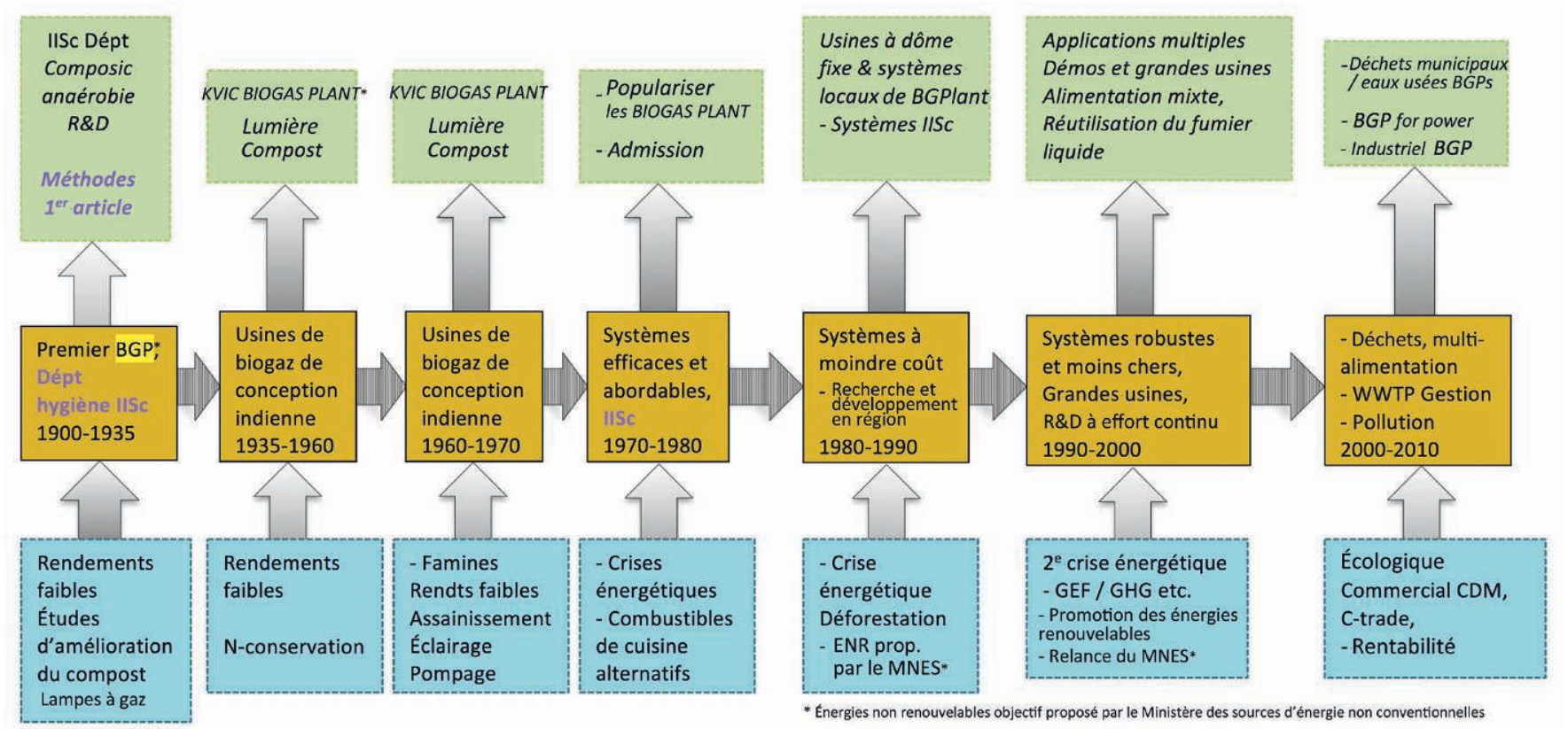

5. Ce schéma représente les différents facteurs qui ont influencé la recherche-développement sur le biogaz en Inde (Chanakya \& Moletta 2005a, op. cit.). Les blocs de la rangée inférieure (en bleu) représentent les facteurs et les problèmes qui ont été étudiés pendant la période concernée, les blocs jaunes montrent les questions de recherche-développement qui ont été examinées (rangée du milieu) et les blocs de la rangée supérieure indiquent les résultats obtenus. Le compte rendu interne semble indiquer que l'objectifétait d'étudier les processus microbiologiques et physico-chimiques sous-jacents, et plus précisément leur potentiel énergétique et leur viabilité, afin de déterminer s'ils pouvaient servir à produire une " énergie durable et accessible à tous ». On reconnaît dans cette figure trois grands domaines : 1) l'étude de la digestion de la biomasse en vue d'améliorer le processus, 2) l'émergence des technologies de digestion des résidus agricoles et de la biomasse, et 3) les questions de gestion durable qui ont été examinées et résolues. Or, même posé en ces termes, le passage à un mode de gestion durable ne privilégie pas une région géographique particulière, mais met en avant les composantes scientifiques qui sont orientées vers une conception mondiale de la digestion de la biomasse. Fait intéressant, la circulation des idées technologiques s'effectue selon deux axes orthogonaux dont les flèches indiquent le mouvement dans une direction. Les flèches d'influence vont dans un seul sens et il n'y a pas de boucle de rétroaction. De surcroît, il y a peu de connexions entre les différents chemins d'évolution parallèles, chacun d'eux (représenté par une colonne) n'étant connecté qu'au chemin précédent, comme dans une chaîne markovienne. A l'évidence, il s'agit d'une représentation bidimensionnelle de l'innovation de ce processus évolutif. (Source : Chanakya \& Malayil 2012 : 111-143) 
en effet à la recherche de technologies et de programmes dans le cadre d'interventions en Afrique francophone (Chanakya \& Moletta 2005a). L'INRA de Narbonne et l'INSA de Lyon s'intéressent donc à ces usines. Les deux instituts espèrent à l'origine faire la démonstration de leur technologie en utilisant des déchets agricoles provenant de serres et de vignobles français. Mais en France, en raison des températures, les chambres de réaction doivent être enterrées, ce qui est interdit par le droit européen en raison du risque de pollution de la nappe phréatique en cas de fissure. Tandis que les programmes se poursuivent en Afrique, dans les vignobles français, les travaux sont menés, depuis 2005, sous l'égide des Projets du Réseau franco-indien des acteurs de l'eau en Inde du Sud (Chanakya \& Moletta 2005b).

Depuis les années 1980, le rendement de la production de gaz a augmenté de $20 \%$. En raison de la défaillance des systèmes civiques urbains, le gaz est désormais utilisé à d'autres fins. Dans l'État du Kerala, les usines de biogaz ne sont plus construites pour répondre aux besoins en gaz et en énergie des communautés, mais pour le traitement in situ des ordures ménagères solides et des déchets agricoles (Johansson et al. op. cit.). C'est comme si nous étions revenus à la situation initiale. Les technologies ont néanmoins évolué, de même que les fonctions qu'elles étaient censées remplir autrefois. L'Inde rurale de Gandhi et ses besoins sont passés au second plan. Au cours des décennies, le biogaz a vu ses usages se multiplier et a investi le discours sur les énergies renouvelables. Il peut désormais jouer un rôle de premier plan dans les débats sur le développement durable, et ses nombreuses applications lui confèrent une place de choix dans la nouvelle économie du méthane: l'excédent de biogaz produit dans les zones rurales peut servir de carburant automobile et les générateurs de biogaz être utilisés pour alimenter des piles à combustible et de petits groupes moteurs. L'utilisation de capteurs de gaz à effet de serre garantissant des émissions exemptes de méthane permet en outre d'atténuer les problèmes liés à ce gaz.

La thèse développée ci-dessus s'appuie sur une brève étude d’un unique arbre généalogique, au sein d'une famille de technologies de biogaz par ailleurs dendrifiée et nettement plus vaste. L'histoire est décrite en détail jusque dans les années 1980, puis les évolutions ultérieures sont signalées afin de montrer comment les technologies, un temps remisées, sont sans cesse tirées de l'oubli. Dans ce travail, nous avons cherché à mettre en évidence la combinatoire de facteurs contingents qui ont joué un rôle constitutif dans le cycle de développement de ces technologies. Parmi ces contingences figurent les ressources (disponibilité des différents types de déchets utilisés pour la biogazification), les contraintes sur les ressources (fourrage en quantité suffisante pendant les mois secs, pénurie de biogaz dans le village de Pura, crise énergétique mondiale), l'existence au moment voulu de compétences techniques, scientifiques et technologiques très variées (maintenance technique au niveau du village, connaissances locales sur les ressources et les topologies, pool de compétences spécialisées mobilisables et susceptibles de réorienter les parcours de recherche-développement), la nature changeante de la « demande sociale» (souvent considérée comme l'élément déclencheur de l'innovation), et les bons choix (critères à utiliser pour choisir la 
technologie). L'environnement de l'usage énergétique a orienté l'application et l'utilisation finale de la technologie, qui a souvent évolué par petites technologies anticipées. Avec l'évolution du paysage de la recherche en ingénierie, les centres et les programmes de recherche ont été rebaptisés pour se conformer aux impératifs de politique publique et aux priorités de financement d'organismes internationaux. D'où l'adaptation de savoir-faire anciens (ingénierie et sciences) et l'appropriation de compétences nouvelles (interdisciplinarité de l'après-révolution infogénétique). Ce remaniement de l'ancien et du nouveau s'est manifesté dans l'évolution technologique en brouillant la frontière high tech.

\section{Notes}

1. Il y a une vingtaine d'années, ASTRA est devenu le Centre pour les technologies durables (Center for Sustainable Technologies).

2. Voir la lettre d'information initialement produite par la revue scientifique Nature et intitulée SciDev, lancée sous la direction du regretté David Dickson; cité en références D. Raina (2016) et UNESCO 2015.

3. Au moment où Reddy fait ses débuts comme électrochimiste, son programme sur les alternatives technologiques attire l'attention des chercheurs sur l'analyse des politiques énergétiques. Ce travail culmine avec la publication de son ouvrage révolutionnaire, Energy for a Sustainable World, co-signé avec trois autres auteurs, qui reçoit le prix Volvo. On peut avancer sans hésiter qu'entre les années 1970 et la fin des années 1990, Reddy est de loin l'un des savants indiens qui ait le plus réfléchi au rapport entre science et société, et influencé de manière significative les mouvements sociaux visant à démocratiser la culture scientifique et à influencer les choix technologiques appliqués au développement. Le programme développé par Reddy se démarque tout particulièrement dans l'univers de certitudes de l'Indian Institute of Science : bien avant que la philosophie des sciences post-kuhnienne n'apporte les outils intellectuels pour contester les hiérarchies de pouvoir et d'influence qui existaient traditionnellement entre disciplines, l'auteur émerge non seulement comme un scientifique dissident, mais comme une figure emblématique du doute.

4. La tension entre la théorie de l'acteur réseau de Bruno Latour et la pensée deleuzienne des assem- blages a été abondamment commentée. Des travaux récents suggèrent que ces deux voies ne seraient pas si opposées entre elles que l'on a pu le croire, même si la première se concentre sur la métaphysique de la présence et la seconde sur la métaphysique des potentialités. Selon cette dernière, les entités existent en rapport avec d'autres entités sans cependant être entièrement déterminées par elles : elles contiennent un surplus qui excède ces relations et leur permet de se connecter à d'autres assemblages.

5. À de très nombreuses reprises, Reddy cite en exergue de ses présentations universitaires ou publiques la remarque de Gandhi selon laquelle la terre produit assez pour satisfaire les besoins de chacun, mais non pour satisfaire leur cupidité - une idée que Gandhi élabore lui-même en ces mots : « Nous aurons toujours parmi nous, comme il y en a toujours eu, des personnes qui voient la poursuite de la richesse comme un but dans la vie. Mais nous avons toujours considéré qu'il s'agit d'un écart par rapport à l'idéal... J'entends beaucoup de nos concitoyens dire que nous arriverons à être aussi riches que l'Amérique mais en évitant ses méthodes. Je voudrais suggérer qu'une telle entreprise, si elle est tentée, est vouée à l'échec. On ne peut être en un même moment "sage, tempéré et furieux" ». (Propos tirés d'une conférence prononcée par Gandhi à Allahabad le 22 décembre 1916, « Economic versus Moral Progress » [Progrès économique versus progrès moral].

6. Gilbert J. Fowler est nommé à la faculté du Département de chimie de l'Indian Institute of Science 
en 1916 et travaillera par la suite comme consultant pour de nombreuses entreprises de l'industrie chimique.
7. Voir les archives de revues scientifiques et de bulletins comme Indian Farming, Khadigramodyog, Farmers Digest, Ind J Agric. Sci., les bulletins spéciaux d'ICAR/IARI, etc.

\section{I'auteur}

Dhruv Raina est professeur à l'université Jawaharlal-Nehru, New Delhi. Il a étudié la physique à l'Institut Indien de Technologie, Mumbai et a obtenu son doctorat de philosophie des sciences à l'université de Göteborg. Sa recherche s'est centrée sur les politiques et les cultures de la connaissance scientifique en Asie du Sud. Il a codirigé Situating the History of Science: Dialogues with Joseph Needham (1999), Social History of Sciences in Colonial India (2007) avec S. Irfan Habib, et Science between Europe and Asia (2010) avec Feza Gunnergun. Il est co-auteur de Domesticating Modern Science (2004) et la publication de ses travaux inclut Images and Contexts : the Historiography of Science and Modernity (2003) et Needham's Indian Network (2015).

Hoysala N. Chanakya est directeur scientifique du Centre des technologies durables à l'Institut Indien des Sciences, Bangalore. Sa recherche porte sur l'évolution des techniques de digestion anaérobie et sur les processus d'économie d'énergie dans les énergies vertes et le recyclage par l'eau. Ses intérêts actuels s'attachent à la réalisation d'un « village en bio-énergie totale », la disparition des décharges urbaines, la réhabilitation d'un lac de ville recyclant des eaux usées, au développement durable des zones rurales. Au long des années, il a ouvert la voie de la planification participative et à l'évaluation des technologies intervenant dans différents contextes ruraux et peri-urbains.

\section{Iconographie}

Image d'ouverture. Réservoir à biogaz installé en Allemagne au milieu des champs. Cette usine pourrait approvisionner environ 1000 foyers en électricité et 150 en chaleur. (CUTS Products GmbH, Germany.

1. (C) myclimate.org

\section{Références}

Abbasi, T., Tauseef, S. M. \& S. A. Abbasi 2012 Biogas Energy. New York : Heidelberg, Londres : Springer : 11-23 (chap. 2 : «A brief history of Biogas »).

Acharya, C. N. 1940a « Composts and soil fertility », Ind. Farming I (2) : 66-68.

— 1940b «Composts and soil fertility», Ind. Farming I (3) : 121-126.

— 1935 « Studies on Anaerobic Decomposition of Plant Materials, parts IV. Decomposition of plant substances of varying composition », Biochemical Journal 29 : 1459-1467.

Chanakya, H. N. \& P. Balachandra 2012 «Enabling Bioenergy Deployment for LCE : Methane for Cooking in India » in A. Srinivasan, F. Ling, S. Nishioka \& Mori, H. Climate smart development in Asia : transition to

2. (C) Amanbagh.org.

3. (C) SKG Sangha / GoodPlanet.

4. Chanakya \& Balachandra, 2012.

5. Chanakya \& Malayil, 2012.

low carbon and climate resilient economies. Londres : Earthscan.

Chanakya, H. N. \& S. Malayil 2012 « Anaerobic Digestion for Bioenergy from Agro-Residues and other Solid Wastes - An Overview of Science, Technology and Sustainability », Journal of the Indian Institute of Science 92 (1).

Chanakya, H. N. \& R. Moletta 2005a « Small scale anaerobic digestion of urban and rural solid wastes-emerging trends in India », Proc. 4th Intl. Symp. On Anaerobic Digestion of Solid Wastes, Copenhagen Aug $31 \mathrm{Sep}$ 2002. IWA $1: 277-284$.

Chanakya, H. N. \& R. Moletta 2005b « Performance and functioning of USW plug-flow reactors in a 3-zone fermentation model » in B. K. Ahring \& Hardtmann, 
H. dir. Proc. 4th IWA Intl. Symp. Anaerobic Digestion, Aug. 31 Sep. 2002. DTU Denmark, vol. 1 : 277-284

Chanakya, H. N., Borgaonkar, S., Rajan, M. G. C. \& G. Meena 1992 «Two Phase Anaerbic Digestion of Water Hyacinth or Garbage », Biores. Technol. 42 : 219-230.

Chanakya, H. N. \& K. Deshpande 1985 [1983] « Sand bed filtration for biogas slurry », Proc. II ASTRA Seminar, 2-3 septembre : 64-68.

Chawla, O. P. 1986 Advances in biogas technology. New Delhi : ICAR

Chawla, O. P., Laura, R. \& M. A. Idnani 1969 « Note on stimulation of anaerobic fermentation of dung in the cowdung-gas plant», Indian 1. agric. Sci. 39 (11) : 1040-1043.

Desai, S. V. \& S. C. Biswas 1945 « Manure and Gas Production by Anaerobic Fermentation of Organic Wastes », Ind Farming VI (2) : 67-71.

Edgerton, D. 2007 Shock of the Old : Technology and Global History since 1900. Londres : Profile Books; New York : Oxford University Press. [traduction française: Ch. Jeanmougin 2013 Quoi de neuf? Le rôle des techniques dans l'histoire globale. Paris : Seuil].

Elzinga, A. \& A. Jamison 1981 «Cultural components in the scientific attitude to nature : Eastern and Western modes », Technology and Culture, Occasional Report Series 2

Forman, P. 2007 « The Primacy of Science in Modernity, of Technology in Postmodernity, and of Ideology in the History of Technology », History and Technology $23(1-2): 1-152$.

Fowler, G. W. \& G. V. Joshi 1921 « Fermentation of Cellulose », J. Indian Inst. Sci. 3 (39)

Gandhi, M. K. 1922 [1916] «Economic versus Moral Progress » Speeches and Writings of M. K. Gandhi with an Introduction by Mr. C.F.Andrews and a Biographical. Madras : G. A. Natesan and Co. : 286$293 ; 290-291$

Gellar, H. S. 1982 « Fuel Efficiency and Performance of Traditional and Innovative Cookstoves », Proceedings of Indian Academy of Sciences, Section C : Engineering Science 5 (4) : 373-393.

Guha, R. 2007 « Mahatma Gandhi and the Environmental Movement » in M. Rangarajan Environmental Issues in India : A Reader. Delhi : Pearsons Longman : 111-128.

Harding, S. dir. 2011 The Postcolonial Science and Technology Reader. Durham (Caroline du Nord) / Londres: Duke University Press.
Idnani, M. A. \& S. Varadarajan 1974 « Preparation of fuel gas and manure by anaerobic fermentation of organic materials », ICAR Tech Bull. 46, Delhi : ICAR.

Johansson, T. B., Kelly, H., Reddy, A. K. N. \& R. H. Williams 1993 Renewable Energy-Sources of Fuels and Electricity. Washington D.C. : Island Press.

Khandelwal, K. C. \& S. S. Mahadi 1986 Biogas Technology: a practical handbook. New Delhi : Tata McGraw-Hill.

McClellan III, J. \& H. Dorn 1999 Science and Technology in World History : An Introduction. Londres/ Baltimore : The Johns Hopkins University Press.

MacGregor, N. 2012 A History of the World in 100 Objects. Londres : Penguin Books.

Müller, M. \& C. Schurr 2016 « Assemblage Thinking and Actor Network Theory: Conjunctions, Disjunctions and Cross fertilisations », Transactions of the Institute of British Geographers 41 : 217-229.

Narashima, R. 2003 « Science, Technology and Society: A Tale about Rocket Development during 1750-1850 » in R. Narashima, J. Srinivasan \& S. K. Biswas dir. The Dynamics of Technology : Creation and Diffusion of Skills and Knowledge. Londres/New Delhi : Sage.

Raghunandan, B. N. \& H. N. Chanakya dir. 2004 Rural Technology - A 25 Years Retrospective, vol. 1 and 2. Indian Institute of Science IISc Bangalore : Centre for Sustainable Technologies.

Raina, D. 2016 « Mainstreaming Indigenous Knowledge: The Genealogy of a Metaconcept » in S. Hodges \& Rao, M. dir. Public Wealth and Private Health : Stem Cells, Surrogates and Other Strategic Bodies. Delhi : Oxford University Press : 252-268.

- 2003 Images and Contexts : The Historiography of Science and Modernity in India. Delhi : Oxford University Press.

— 1997 « Evolving Perspectives on Science and History : A Chronicle of Modern India's Scientific Enchantment and Disenchantment », Social Epistemology 11 (1): 3-24.

— 1993 «The Technological Determinism Embodied in a Development Research Programme : Or Doing Appropriate Technology in a Big-Science/High-Tech Environment », Journal of Scientific and Industrial Research 52 (juillet) : 471-482.

Raina, V., Chowdhury, A. \& S. Chowdhury 1997 The Dispossessed : Victims of Development in Asia. Hong Kong : Arena Press. 
Rajabapaiah, P., Chanakya, H. N. \& A. K. N. Reddy 1981 «Interim Report Further Studies on Biogas Technology ». CST, Technical Report. Indian Institute of Science : Bangalore.

Rajabapaiah, P., Ramanayya, K.V. Mohan, S. R. \& A. K. N. Reddy 1979 « Studies in biogas technology Part 1 : Performance of a conventional biogas plant », Proc. Ind. Acad. Sci. 2 (3) : 357-363.

Reddy, A. K. N. 1978a « What is an Appropriate Technology? » Mimeograph (Bangalore).

- 1978b «Technologies Appropriate to Rural Development », INSA Seminar on Contributions of Science to Rural Development, New Delhi.

Reddy, A. K. N. \& K. Prasad 1997 « Technological alternatives and the Indian energy crisis », Economic and Political Weekly 9 (33-34) : 1465-1502.

Reddy, A. K. N., Goldemberg, J., Johansson, Th. B. \& R. H. Williams 1988 [1987] Energy for a Sustainable World. [World Resources Institute] New Delhi : Wiley-Eastern.

Reddy, A. K. N. \& D. K. Subramanian 1979 « The design of rural energy centres », Proceedings of the Ind. Acad. of Sci. X (2) : 395-416.

Sathianathan, M. N. 1975 Biogas : Achievements and Challenges. New Delhi : Assoc. Voluntary Agencies in Rural Dev. (AVARD).
Schumacher, E. F. 1975 Small Is Beautiful. New York : Harper Perennial Books.

Serres, M. \& B. Latour 1990 Conversations across Science, Culture and Time, translated by Roxanne Lapidus, University of Michigan Press : Ann Arbor. [première édition française : Serres, M. 1992 Éclaircissements. Cinq entretiens avec Bruno Latour. Paris : Éditions François Bourrin].

Seshadri, C.V. 1999 The Evolution of Appropriate Science. Rural Technology. A 25 Years Perspective (vol. 1) Centre for Sustainable Technologies, IISc Bangalore : 97-110.

Smith, J. 2009 Science and Technology for Development : Development Matters. Londres/New York : Zed Books.

Subbarayappa, B. V. 1992 In Pursuit of Excellence : A History of the Indian Institute of Science. Delhi : Tata McGraw-Hill.

UNESCO 2015 UNESCO Science Report : Towards 2030. Paris : UNESCO.

Visvanathan, S. 1997 A Carnival for Science : Essays on Science, Technology and Development. Delhi : Oxford University Press.

— 1985 Organizing for Science. New Delhi : Oxford University Press.

\section{Traduction}

La rédaction de TechniquesECulture tient tout particulièrement à remercier Olivier Morice et Valentine Leÿs pour la qualité et la rigueur de leur travail d'adaptation à la langue française de cette étude très pointue sur les biogaz.

\section{Pour citer l'article}

D. Raina, D. \& H. Chanakya 2017 «Une histoire des contingences technologiques. Digestion anaérobie et évolution de la recherche sur la technologie du biogaz», TechniquesECulture 67 « Low tech? Wild tech! », p. 154-175. 